\title{
Towards evidence-informed sports safety policy for New South Wales, Australia: assessing the readiness of the sector
}

Policy-related interventions can contribute significantly to successful public health action. ${ }^{1}$ However, inadequate health promotion policy can result in: poor stakeholder engagement; responsibility division across government; short-term funding and unsustainable programmes; haphazard resource development and distribution; mixed messages; information overload; and a lack of standardised guidelines. ${ }^{2}$

The need for safety policy in sport is well recognised, and there has been a recent call for global attention to the development of sports safety policy to ensure significant safety gains are made for all. ${ }^{3}$ This call has highlighted the need to better integrate the efforts of governments (particularly health departments) and sporting organisations, and to improve the sports safety "science to policy" interface. There are few examples of the development of national and state/ provisional overarching sports safety policies, regulations, or strategic plans. In Quebec, Canada, the government adopted the Act Respecting Safety in Sport in 1979 and established the Quebec Sports Safety Board with responsibility for "supervising personal safety and integrity in the practice of sports". ${ }^{4}$ The Quebec Sports Safety Board coordinates provincial sports safety activities, particularly focusing on developing and implementing safety standards in partnership with sports federations. In New Zealand, the Accident Compensation Corporation has established the SportSmart national sports injury prevention programme to systematically reduce and eliminate avoidable injuries by implementing and monitoring specific strategies. ${ }^{5}$

In Australia, sports injury prevention has been on the national health policy agenda to varying degrees since $1994,{ }^{6}$ but policy progress has been limited, and primarily focused on "micro policy" covering single issues (eg, drugs in sport, pregnancy, portable soccer goals, etc) ${ }^{7}$ or specific to individual sports, such as football codes. ${ }^{9} 10$

In 1995, the Australian Sports Injury Prevention Taskforce was established, leading to the development of the Australian Sports Safety Framework in 1997, ${ }^{11}$ and the establishment of a lead national agency, SportSafe Australia. This agency represented a strong health and sports sector partnership, but it focused mainly on resource development and dissemination, and undertook little policy-based work. ${ }^{12}$ Its Federal Government funding was withdrawn in 2000. To our knowledge, there has been no systematic consideration of an Australian "macro" sports safety policy response since the Australian Sports Safety Framework.

According to Kingdon, three criteria must converge for an issue to be placed on the public (or sports sector) agenda, enabling the policy process to begin: there must be the perception that the issue is a problem requiring action to resolve it (problem stream); there must be an identified solution to the problem (policy stream); and there must be support, such as a change in public opinion or pressure for action from key stakeholder groups (political stream). ${ }^{13}$ In this study we aimed to explore, with reference to Kingdon's three streams, the potential to develop a state-wide sports safety policy for the most populous state in Australia, namely New South Wales. In conceiving this project, policy was defined as "a broad statement of goals, objectives and means that create the framework for activity". ${ }^{14}$ As a framework for policy analysis, we used the policy analysis triangle ${ }^{15}$ which has been successfully used in the health sector, but has wider application. This approach seeks information on contextual opportunities and constraints to policy (situational, structural, and cultural), the process of policy making (such as agenda setting, formulation, and implementation), and the content of policy. ${ }^{14}$ It seeks to understand how these factors are influenced by, and interact with, the needs and roles of significant stakeholders (policy actors). ${ }^{14}$

This paper outlines the current sports safety policy and planning activities in place in New South Wales, and describes the perceived barriers, potential motivators, and enablers, to sports safety "macro" policy development in this state.
It provides background to inform strategies and tactics that may influence future policy process in terms of agenda setting, policy formulation, and implementation.

\section{METHODS}

Three complementary data collection activities were undertaken: an industry survey and semi-structured interview to provide an overview of existing safety policy and planning activities in place; a discussion forum with representatives of state sporting organisations (SSOs), government departments, and non-government organisations (NGOs); and a roundtable meeting with a small number of key stakeholders. An iterative approach was taken where each latter stage was informed by previous stages. The survey and interview responses were collated and presented at the discussion forum; a report summarising the outcomes of the discussion forum informed the roundtable meeting.

Australian organised sport is generally administered hierarchically. National sporting organisations (often affiliated with international sports bodies) provide strategic, policy, and operational guidance to SSOs who in turn oversee regional and community activities. As this study targeted the state level, a sample of SSOs was invited to participate in a written survey and telephone interview. The survey, based on the Australian Sports Safety Framework ${ }^{11}$ and a previously validated questionnaire, ${ }^{16}$ asked about the organisations' current safety policies. The semi-structured interview asked about safety planning and promotion activities; areas of sports safety need or difficulty; and perceived advantages or disadvantages of a state-wide sports safety policy.

The selected SSOs represented New South Wales-based sports with a large number of registered participants, ${ }^{17}$ sports with a high number of injuries, ${ }^{18}$ and/or selected sports for people with a disability. Sports were identified from the State Government's Department of Sport and Recreation directory. Additional alternative representatives of selected sports were identified when the initial contact was unsuccessful. Of the $38 \mathrm{SSO}$ s invited to participate, 19 completed the survey and 21 completed the interview (23 SSOs participated in one or both).

During the second phase, the selected SSOs and state government departments and state-based NGOs considered by the research team to have a role in sports safety, were invited to a discussion forum to explore perceived barriers, enablers, and motivators to developing a state-wide 
sports safety policy. Twenty-six representatives from 23 organisations (11 SSOs including two SSO membership bodies, five government departments, and seven NGOs) attended. Participants represented the sport, health, school education, product safety, youth advocacy, child safety, sports insurance, sports regulation, and sports injury research sectors.

In the third phase, forum participants were invited to a roundtable meeting to discuss a process for developing a statewide sports safety policy. Participants included representatives from: the Government's Department of Sport and Recreation; a state statutory community sports insurance and safety promotion organisation (unique to New South Wales); the state branch of the national sports medicine and sports science representative body; and a membership body representing a range of sporting organisations.

To ensure the forum and meeting reports were an accurate reflection of events, draft reports were circulated to participants for validation.

The Medical and Community Human Research Ethics Advisory Panel of the University of New South Wales approved this project.

\section{OUTCOMES}

\section{Contextual opportunities and constraints}

The 19 surveyed SSOs reported having various safety policies in place. Government mandated policies (eg, child protection) were reported by all; and most reported having policies central to playing the sport (eg, coaching standards, rules, protective equipment). Safety policies indirectly related to the sport (eg, pregnancy, infectious disease control) were less frequently reported. Around three-quarters of SSOs reported gathering injury data; one-third reported having a safety coordinator/committee; fewer than onequarter reported having safety as a regular agenda item at board/committee meetings, and/or a dedicated safety budget.

The 21 interviewed SSOs reported mixed approaches to identifying injury risks. Proactive approaches were uncommon, but included safety audits and analysis of injury/insurance data. The SSOs obtained information about safety strategies from several sources, including national sporting organisations, relevant organisations (eg, a sports medicine authority), the Government's Department of Sport and Recreation, and board/council members with specialist knowledge.

In general, respondents believed their sports safety plans could be enhanced by improving their comprehensiveness, dissemination, or implementation.

Several barriers to sports safety policy development and implementation were identified (see table 1). Sports safety was given an apparent low priority at the state government level, as key government department stakeholders did not report this as an existing policy focus. No current state policy champion was identified, and no state-level, sports safety partnership was evident. The SSOs reported capacity limitations towards developing and implementing organisation-wide safety policy, including: staffing and expertise; financial; data; and organisational management structure limitations. Moreover, the nature of community sport created difficulties with mandating, communicating, and monitoring "grassroots" safety activities. The remoteness of rural clubs was also identified as a barrier.

Table 1 presents the identified motivators and enablers for successful state-wide sports safety policy development and implementation. Support from representative bodies (including high profile SSOs and national sporting organisations) and other significant stakeholders (such as health, education, sports medicine, local government), and credible leadership (eg, the

Table 1 Identified barriers, motivators, and enablers to sports safety policy in New South Wales, Australia

Barriers
Apparent low priority of sports safety at all levels*
Current lack of state-level leadership*
Current lack of support at the state level*
Insufficient SSO and community organisational
infrastructure*
Limited SSO and community sporting organisational
capacity for sports safety (including limited finances,
people, time, expertise, and access to data) †

people, time, expertise, and access to data) +

The volunteer nature of "grassroots" sports administration $†$

Lack of control over facilities and environments $†$

The geographic remoteness of rural clubs and associations $†$

Difficulties in achieving effective dissemination of information to all levels of sport $†$

Lack of incentives to encourage uptake

The very different nature of injury and injury risks across different sports $\dagger$

${ }^{*}$ Conclusion drawn by the research team after all phases of the research.

Motivators and enablers

Reframing sports safety to appeal to the "drivers" for action of the various stakeholders*

A credible state-level leader $\ddagger$

Widespread support by representative bodies, including SSOs, non-government organisations, and government departmentsł

Capacity building and resourcing to improve SSO and community organisational infrastructure*

SSOs and community sporting organisations with the capacity for safety promotiont; training and education essential $\neq$; make it integral to core business so that it is ongoing and sustainable $\underset{\uparrow}{\ddagger}$ integrate into existing club development and risk management initiatives $\ddagger$

Consumer (sports participants and parents of children) demand for "safe sport" $\$$

Involvement of representatives from facility managers (eg, local government) in policy development* Specific strategies to target rural and remote regions*

Resources to support safety at all levels-finance, training, information:; use of effective communication strategiesł; "train the trainer" approachł; use of established networks

Accountability for, and mandatory application of directives $\Phi$; links to accreditation $\ddagger$

Flexibility to enable adaptation to a range of sports; based on universally relevant and applicable principles $\underset{\ddagger}{\ddagger}$

†Reported by SSOs during telephone interview.

tIdentified at the discussion forum.

SIdentified at the roundtable meeting.

qIdentified at the discussion forum as a means of making policy work, but not favoured by SSOs. 
item on board agenda, etc); a comprehensive checklist of safety issues (protective equipment, playing environment, infectious disease, child protection, etc) from which sports could select those of relevance; practical tools/resources to help sports identify, prioritise, manage, and monitor unacceptable risks, and which could be adapted to their specific needs and context (injury surveillance templates, sample policy statements, etc); case studies illustrating successful implementation of safety policy; and sources of additional information and resources.

It was generally agreed that the Sports Safety Guidelines should be incorporated into existing safety, risk management, and administration initiatives. Moreover, they should be linked to existing training and resources to reduce duplication.

In addition, the roundtable meeting participants identified the need for a "strategic vision" type document (hereafter Sports Safety Framework) to outline why action is needed, what action should be taken, and how it should be done. This should include information about partners, funding, outcomes, evaluation, and links with other government and nongovernment strategies. Such a document would enable these agencies to make work in sports safety more legitimate and easier to incorporate into ongoing core business. The SSOs expressed little interest in a Sports Safety Framework document during the discussion forum.

\section{Policy process}

The policy development recommendations, which were supported at the roundtable meeting, included establishing and resourcing a multi-agency partnership of key policy actors (hereafter Sports Safety Reference Group) to: develop the Sports Safety Framework; oversee a smaller representative working group to develop the Sports Safety Guidelines; coordinate the dissemination of the Sports Safety Guidelines, using existing networks; and build SSO capacity to adopt a strategic, evidenceinformed and sustainable approach to safety. The Sports Safety Framework and Guidelines together represent the state-level sports safety "policy" response generally supported by participants.

Table 2 summarises the recommended actions and strategies to address the barriers and create the motivators and enablers to sports safety policy development and implementation identified during this research.

\section{DISCUSSION}

The rationale for developing and implementing national or state-level injury prevention policy is to obtain commitment and to coordinate "the efforts, roles, responsibilities, and resources of the many actors involved" ${ }^{19}$ This study explored the potential to construct macro-level sports safety policy through discourse and mobilisation of key policy actors to generate a shared understanding of the injury problem and appropriate responses, and develop ownership and a common point of reference which would sustain a strategic and coordinated approach to safety.

This study identified the presence of numerous micro-level policies and processes to promote safety in sport within SSOs. The absence of macro-level sports safety policy appears to have fostered a fragmented, reactive, individualissue based approach to safety dependent on individual organisational capacity, rather than a coordinated, proactive, and strategic approach based on sector or industry commitment.

Applying Kingdon's prerequisite for the convergence of three streams for the public policy process to begin, ${ }^{13}$ it is clear that the requirement of the problem stream has not been previously fulfilled. Although individual safety issues have been identified by government and SSOs as important enough to warrant micro-level policy action, the broader issues of sports injury prevention and safety have not yet been perceived as problems requiring macrolevel policy action to resolve. However, there was general agreement among participants in this study that, although

Table 2 Actions and strategies to address barriers and promote motivators and enablers to sports safety policy development and implementation for New South Wales, Australia

Action Strategy

Identify a credible leader

Gain widespread support

Build capacity, create supportive organisational infrastructure, and develop sustainability

Produce universally applicable material

Generate a demand for safe sporting opportunities Empower volunteers

Disseminate effectively

Address geographic remoteness
Government's Department of Sport and Recreation is a credible leader $\dagger$

Include all significant stakeholders on the Sports Safety Reference Group $\dagger$

Gain endorsement of the Sports Safety Guidelines by all respected stakeholders $†$

Reframe "safety" so that it appeals to the drivers in each key stakeholder group; eg, for sporting organisations this may be improvement in team performance, reduced risk of successful litigation, higher participation levels, rather than the "cost of injury"*

Provide educational and training opportunities. † Trainers should have expertise in safety-related risk management, not just legal or insurance expertise. * Training should include support for organisational change and establishing the required organisational management structure (leadership, commitment, communication, designated responsibility, regular review, resourcing/budget, regular item on agenda, etc) to ensure safety becomes "core business" *

Align training with existing resources and programmes, including risk management programmes such as those already being delivered by the Government's Department of Sport and Recreation†

Link adoption of Sports Safety Guidelines to funding from, and reporting to, the Government's Department of Sport and Recreation*

Advocate for the Government's Department of Health and the insurance industry to provide timely and relevant sports injury data* $^{*}$

Address all the capacity building requirements to promote ongoing implementation and long-term sustainability*

Sports Safety Guidelines should be process-oriented and enable sporting organisations to adapt them to suit their needs. They should not specify mandatory or prescriptive micro-level safety policies to be adopted by all $\dagger$

Educate sport participants and parents of participants about safety, and how to identify safe clubs. Use existing networks $\dagger$ Sports Safety Guidelines should be quick and simple to use. Provide tools, resources, and templates to lessen the workload. Provide case studies by way of example, and guidance on where to go for further information $\dagger$

Invite all key stakeholders to champion the Sports Safety Guidelines to their members $†$

Use the existing communication networks of all key stakeholders to disseminate the Sports Safety Guidelines $†$ Ensure specific needs of regional and rural providers are identified and addressed*

Cldentified during the survey, interview, discussion forum, or roundtable meeting.
the 
some SSOs have greater capacity than others to identify, develop, and implement safety initiatives, the sports sector would welcome assistance and guidance to develop a strategic, evidence-informed and sustainable approach to safety. It was also identified that framing the issue of injury differently for different policy actors may be a potential way of developing traction in the problem stream. Engagement of policy actors could be enhanced by identifying the drivers for each that will facilitate their viewing the issue of injury as a problem that needs to be resolved. For example, the health sector may be more likely to view sports injury as a problem if it is framed in the context of costs to the health service and impact on chronic diseases such as obesity and arthritis. ${ }^{20}$ For the sports sector it may be necessary to frame the issue in relation to impacts on market share, participation rates, public perception, legal and insurance responsibilities, and maximising participant performance.

Importantly, within Kingdon's policy stream, ${ }^{13}$ this project has highlighted that the sports safety policy needs and interests of, and solutions for, sporting organisations are quite different from those of government departments and NGOs. The supported policy solution included one component directed at government departments and NGOs outlining the agreed policy "strategic vision", alongside other components directed at SSOs, to support the practical application of policy and the implementation of evidenceinformed interventions. Understanding and meeting the various policy needs of different policy actors is vital to the successful translation of sports injury prevention research into effective safety policy and practice. ${ }^{21}$

While it was identified that the development of the strategic vision (Sports Safety Framework) may be possible within existing key stakeholder capacity, it was also acknowledged that the development, dissemination, implementation, and evaluation of the Sports Safety Guidelines would require significant, additional, ongoing funding and commitment to build the capacity essential to create sustainability. The proposed Sports Safety Reference Group, with its multi-sectoral representation, would be ideally placed to advocate for sustained government funding to promote this.

To meet the requirements of Kingdon's political stream, ${ }^{13}$ a credible policy champion needs to be identified to coordinate and drive the process, and significant policy actors need to be engaged. While the Government's Department of Sport and Recreation was identified as the most credible leader, several other actors were identified as necessary supporters and partners in the policy-making process because of their knowledge, resources, expertise, and jurisdictional influence. For example, the Government's Department of Health was identified as an important partner by participants because they perceived it could provide funding, injury data and health promotion/injury prevention expertise. Other agencies, such as local government, were considered essential because they were perceived to manage specific aspects of the problem and solution (eg, sporting facilities), which were beyond the direct control of the sports sector. The sports medicine professional body was identified as a source of expert advice, particularly in relation to the development of the Sports Safety Guidelines. Participants considered a multi-agency partnership essential to developing the political environment required to achieve sports safety policy success, as has been highlighted in other health promotion policy development contexts. $^{22}$ However, we detected that there may be a disparity between the actual level of engagement of some stakeholders, and that expected of them by other stakeholders. This could be a barrier to significant state level progress, and should be monitored. Social pressure can also be an important political driver for policy action. Community sports consumers and parents of sports participants are a potentially powerful group from which demand for policy that results in safer sporting opportunities could be generated from "the ground up".

In interpreting the findings of this study, several significant limitations are acknowledged. First, the survey and interviews drew on self-reported policies and processes, and is therefore subject to reporting bias. Second, the evidence-base on which reported policy and practice were grounded was not assessed. Third, the findings reflect the actions and views of the participants, and not all invited stakeholders participated, which may have reduced the representativeness of the findings. Finally, this project was researcher driven so the extent to which the findings and recommendations arising from this research are acted on will depend on factors outside the research sphere, such as the capacity and continuing motivation of key policy actors and available funding.

\section{CONCLUSION}

Although sports injuries have been identified as a significant public health problem in Australia, ${ }^{23}$ macro-level sports safety policy has not featured prominently on the agenda of Australian governments or the sports sector, as in many other countries, and this is an issue of international concern. ${ }^{3}$ There are many challenges for population-focused sports safety policy development, but this study provides some guidance towards a feasible state-level policy solution. It has also identified the importance of raising public and sports sector awareness and demand, and the reframing of sports safety in terms likely to enhance "problem" identification by all key policy actors. For New South Wales, this study has identified the critical requirements for sports safety policy action and potential ways of meeting them. The time is now ripe to harness the commitment and interest of key policy actors to develop and deliver effective state-level sports safety policy that leads to the widespread adoption and implementation of evidence-informed sports injury prevention interventions.

Acknowledgements The authors wish to thank the many SSOs, the government departments and NGOs who participated in this project; Dr Rebecca Dennis for her input into the initial research proposal; and Dr Jane Elkington who conducted, and assisted in the analysis of, the survey and interview component.

\section{R Poulos, ${ }^{1}$ A Donaldson ${ }^{2}$ C Finch $^{2}$}

${ }^{1}$ The School of Public Health and Community Medicine, The University of New South Wales, Sydney, Australia; ${ }^{2}$ School of Human Movement and Sport Sciences, University of Ballarat, Mt Helen, Australia

Correspondence to Dr R Poulos, The School of Public Health and Community Medicine, The University of New South Wales, Sydney, 2052, Australia;

r.poulos@unsw.edu.au

Funding This research was supported by a grant from the NSW Sporting Injuries Committee. RGP was supported by a National Health and Medical Research Council (NHMRC) Capacity Building Grant in Injury, Trauma and Rehabilitation. CFF was supported by an NHMRC Principal Research Fellowship.

Competing interests None.

Ethics approval Ethics approval was obtained.

Provenance and peer review Not commissioned; externally peer reviewed.

Accepted 28 July 2009

Injury Prevention 2010; $\mathbf{E : 1 - 5 .}$ doi:10.1136/ip.2008.021386

\section{REFERENCES}

1. Schmid T, Pratt M, Howze E. Policy as intervention: environmental and policy approaches to the prevention of cardiovascular disease. Am J Public Health 1995;85:1207-11. 
2. Raupach J, Rogers W, Magarey A, et al. Advancing health promotion in Australian general practice. Health Educ Behav 2001;28:352.

3. Timpka T, Finch CF, Goulet C, et al. Meeting the global demand of sports safety. The intersection of science and policy in sports safety. Sports Med 2008;38:795-805

4. Régnier G, Goulet C. The Québec Sports Safety Board: a governmental agency dedicated to the prevention of sports and recreational injuries. Inj Prev 1995:1:141-5.

5. Accident Compensation Corporation. SportSmart: New Zealand Government. http://www. acc.co.nz/injury-prevention/sport-safety/ acc-sportsmart/index.htm laccessed 21 November 2008).

6. Commonwealth Department of Human Services and Health. Better health outcomes for Australians: national goals, targets and strategies for better health outcomes into the next century. Canberra: Commonwealth Department of Human Services and Health, 1994.

7. Stewart B, Nicholson M, Smith A, et al. Australian sport: better by design? The evolution of Australian sport policy. Oxford: Routledge, 2004.
8. Hooper SL, Macdonald D, Phillips M, eds. Junior sport matters: briefing papers for Australian junior sport. Belconnen, ACT: Australian Sports Commission, 2007.

9. Australian Football League. Laws of Australian football. Australian Football League, 2008.

10. Australian Rugby Union. Australian Rugby Union safety directives for players, coaches, administrators \& match officials. Australian Rugby Union, 2007.

11. Finch C, McGrath A. SportSafe Australia: a national sports safety framework. A report prepared for the Australian Sports Injury Prevention Taskforce. Canberra: Australian Sports Commission, 1997.

12. Department of Health and Ageing. Sports safety in Australia. An update. July 2003. Canberra: Australian Government Department of Health and Ageing, 2004.

13. Kingdon J. Agendas, alternatives, and public policies, 2nd edn. New York: Longman, 2003.

14. Buse K, Mays N, Walt G. Making health policy. Milton Keynes: Open University Press, 2005.

15. Walt G, Gilson L. Reforming the health sector in developing countries: the central role of policy analysis. Health Policy and Plan 1994;9:353-70.

16. Donaldson A, Hill T, Finch C, et al. The development of a tool to audit the safety policies and practices of community sports clubs. J Sci Med Sport 2003;6:226-30.

17. Standing Committee on Recreation and Sport. The exercise, recreation and sport survey. Annual report 2005. Canberra: Australian Sports Commission, 2006.

18. Boufous S, Dennis R, Finch C. A profile of hospitalisations and deaths due to sport and leisure injuries in New South Wales, 2000-2004. Sydney: University of New South Wales, 2006.

19. Sethi D, Waxweiler R, Racioppo F. Developing a national policy for injury and violence prevention. Int J Inj Contr Saf Promot 2008;15:53-5.

20. Finch CF, Hayen A. Governmental health agencies need to assume leadership in injury prevention. Inj Prev 2006;12:2-3.

21. Goulet $\mathbf{C}$. The challenges of adapting theory to practice. J Sci Med Sport 2003;6:139-40.

22. Bellew B, Schöeppe S, Bull FC, et al. The rise and fall of Australian physical activity policy 1996-2006: a national review framed in an international context. Aust New Zealand Health Policy 2008;5:18.

23. Finch C, Cassell E. The public health impact of injury during sport and active recreation. J Sci Med Sport 2006:9:490-7. 